\title{
Projekt „Neue Seidenstraße“: Möglichkeiten der Zusammenarbeit zwischen der OSZE und China
}

\author{
Stefan Wolff und Stephanie Liechtenstein"
}

\section{Zusammenfassung}

Welche Konsequenzen hat Chinas wachsender Einfluss in der OSZE-Region? Am Beispiel der „Neuen Seidenstraße“ wird der chinesische Einfluss in den OSZE-Teilregionen Zentralasien, Südkaukasus/Osteuropa und Westbalkan untersucht. Viele OSZE-Teilnehmerstaaten haben bereits bilaterale Verträge mit China geschlossen. China geht es bei der „Neuen Seidenstraße“ vor allem um Transportkorridore nach Europa, aber auch um den Zugang zu Energie und Ressourcen. Dies hat zwangsläufig geopolitische und geoökonomische Konsequenzen, denen sich die OSZE stellen muss. Was China und die OSZE verbindet, ist ein gemeinsames Interesse an Stabilität und Sicherheit in der Region. Darauf aufbauend könnte die OSZE eine Kooperation mit China anstreben. Dieser Beitrag macht Vorschläge, wie sich die OSZE China annähern und mit China kooperieren kann.

\section{Schlagworte}

China, Belt and Road Initiative, Neue Seidenstraße, OSZE, Konnektivität, Geopolitik.

Bitte zitieren als: Stefan Wolff und Stephanie Liechtenstein, Projekt „Neue Seidenstraße“: Möglichkeiten der Zusammenarbeit zwischen der OSZE und China, IFSH (Hrsg.), OSCE Insights 2/2021 (Baden-Baden: Nomos, 2022), https://doi.org/10.5771/9783748911449-02

\section{Einleitung}

Die „Belt and Road Initiative“ (BRI) Chinas hat sich seit ihrer Gründung 2013 zu einem globalen Handels- und Infrastrukturprojekt entwickelt. Die Initiative,

* Stefan Wolff

Universität Birmingham

Großbritannien

s.wolff@bham.ac.uk

Stephanie Liechtenstein

Freie Journalistin, Wien

office@stephanieliechtenstein.com die Asien, Europa und Afrika entlang der historischen Seidenstraße verbinden soll, hat inzwischen geopolitische und geoökonomische Auswirkungen. Chinas Präsident Xi Jinping sagte bei seiner Eröffnungsrede an der kasachischen Nazarbajew-Universität, dass ein „Silk Road Economic Belt" gemeinsam von China und dessen Partnern errichtet werden solle, „um die wirtschaftlichen Beziehungen und die gemeinsame Kooperation zu vertiefen und den Handlungsspielraum für Entwicklung zu erweitern“. 1 
Im März 2015 gab die Nationale Entwicklungs- und Reformkommission Chinas weitere Details bekannt. Sie definierte die Ziele der auch als „Neue Seidenstraße" bezeichneten Initiative folgendermaßen: „Förderung der Konnektivität zwischen Asien, Europa und Afrika sowie deren angrenzende Gebiete, Stärkung der Partnerschaften der Länder entlang der Neuen Seidenstraße, Errichtung umfassender, mehrschichtiger und verketteter Konnektivitätsnetzwerke sowie Umsetzung einer diversifizierten, unabhängigen, ausgewogenen und nachhaltigen Entwicklung in diesen Ländern. “2

Diese Ideen und Ziele gab es vorher schon, aber nun wurden sie in einem Projekt gebündelt und zu einer Strategie zusammengeführt. 2017 wurde die Initiative in die Verfassung der kommunistischen Partei Chinas aufgenommen - und damit zu einem wesentlichen Teil des chinesischen Staates und dessen Politik. ${ }^{3}$

Die BRI wird heute als das Kernstück der Außen- und Wirtschaftspolitik Chinas angesehen. Die Initiative hat sich mittlerweile zu einem umfassenden, strategischen Instrument für Chinas Führungsriege entwickelt. Sie bringt die geopolitischen und geoökonomischen Ansprüche einer selbstbewussten und entschlossenen Weltmacht zum Ausdruck.

Im Januar 2021 beteiligten sich insgesamt 140 Staaten und 30 internationale Organisationen an der BRI, mit Projekten in über 70 Ländern. Von den 57 OSZE-Teilnehmerstaaten haben mehr als die Hälfte Absichtserklärungen mit China über eine Teilnahme an der BRI unterzeichnet. $^{4}$
Die „Neue Seidenstraße“ sollte zunächst China und Europa über Handelsrouten auf dem Land (Silk Road Economic Belt) und zur See (Maritime Silk Road) verbinden. Heute umfasst sie viel mehr: Es gibt einen weiteren Seeweg über die Arktis (polare Seidenstraße), eine digitale Seidenstraße, entlang derer China in die Digitalwirtschaft investiert, sowie eine "Gesundheitsseidenstraße" (Health Silk Road) für die grenzübergreifende medizinische Zusammenarbeit. ${ }^{5}$

Die Kosten für China sind immens. Einer Kalkulation von PricewaterhouseCoopers aus dem Jahr 2016 zufolge belaufen sich die Kosten der BRI für den chinesischen Staat auf bis zu eine Billion US-Dollar, die in den nächsten zehn Jahren ins Ausland fließen wird. ${ }^{6}$ Bis Ende 2020 wurden davon bereits knapp 94 Milliarden US-Dollar für Investitionen und Bauverträge innerhalb der drei OSZE-Teilregionen ausgegeben: Zentralasien (55 Milliarden US-Dollar), Südkaukasus und Osteuropa (21 Milliarden USDollar) sowie Westbalkanstaaten (18 Milliarden US-Dollar). ${ }^{7}$

Die OSZE kann Chinas Stellenwert und seine zunehmenden Aktivitäten in der OSZE-Region sowie in den angrenzenden Staaten nicht länger ignorieren. Diese haben Auswirkungen auf die OSZE, auf die Beziehungen der OSZE-Teilnehmerstaaten untereinander wie auch auf deren Beziehungen zu China. Dies zeigt sich insbesondere in den drei erwähnten OSZE-Teilregionen, in denen Russland und der Westen traditionell um Einfluss wetteifern. Sie bieten dort rivalisierende Integrationsprojekte 
an: die Europäische Union (EU) und die Eurasische Wirtschaftsunion.

Die BRI strebt derzeit (noch) keine institutionalisierte Integration an. Sie stellt aber dennoch eine mögliche Alternative dar. Die BRI könnte langfristig sowohl ergänzend als auch konkurrierend zur EU und zur Eurasischen Wirtschaftsunion auftreten. Das würde weitere Herausforderungen für die OSZE mit sich bringen, insbesondere bei der Umsetzung des Mandats der umfassenden Sicherheit. Zudem sind die Beziehungen zwischen Russland und dem Westen derzeit so schlecht wie zuletzt am Ende des Kalten Krieges.

Um die bisherigen Auswirkungen der BRI auf die OSZE abschätzen zu können, werden wir im Folgenden die Aktivitäten Chinas in den OSZE-Teilregionen Zentralasien, Südkaukasus/Osteuropa sowie Westbalkan analysieren. Daraus lassen sich voraussichtliche Entwicklungen ableiten und Empfehlungen formulieren, wie die OSZE in Zukunft mit China und der BRI kooperieren könnte. ${ }^{8}$ Eine solche Kooperation sollte prinzipientreu, pragmatisch und strategisch sein, damit die Integrität der Organisation gewahrt bleibt und die OSZE-Teilnehmerstaaten ihrer „Verpflichtung zum Prinzip der umfassenden, kooperativen, gleichwertigen und unteilbaren Sicherheit"9 nachkommen können.

\section{Chinas Aktivitäten in Zentralasien}

In Zentralasien zeigen sich viele der Hauptantriebsfaktoren der BRI. Die Region ist für die Anbindung Chinas an die europäischen Märkte entscheidend, vor allem über Straßen- und Eisenbahnnetze. Diese stellen strategisch wichtige Alternativen zu den Seewegen dar. Die wirtschaftliche Entwicklung und Stabilität Zentralasiens hat für China aber auch eine politische Dimension. Damit will das Land die Sicherheit in der angrenzenden Provinz Xinjiang verbessern, wodurch sich auch negative Spillovers aus Afghanistan leichter verkraften ließen. ${ }^{10}$ China will in dieser OSZE-Teilregion aber nicht nur Transportkorridore errichten, sondern es geht dem Land auch um den Zugang zum Energie- und Rohstoffsektor wie auch um Agrarprodukte für den heimischen Markt.

Kasachstan erhält den Großteil der chinesischen Investitionen. Ungefähr zwei Drittel der chinesischen Geldmittel für Zentralasien gingen seit 2005 nach Kasachstan. Das Land verfügt über eine sehr gut ausgebaute Infrastruktur und ist politisch relativ stabil. Außerdem kann es einen nationalen Entwicklungsplan vorweisen, der eng mit der BRI abgestimmt ist. Die beiden Länder sind wirtschaftlich eng verflochten. China hat massiv in die Ölproduktion Kasachstans investiert. Chinesische Firmen kontrollieren nun rund 25 Prozent dieses Sektors. Des Weiteren kommen ungefähr 75 Prozent aller chinesischer Uranimporte aus Kasachstan. ${ }^{11}$

Der Einfluss der EU und der USA ist in Zentralasien relativ gering. Das ermöglicht es China, diese Region in die eigene Einflusssphäre einzubeziehen. Während Russland nach wie vor der Hauptgarant für Sicherheit in der Region ist, entwickelt sich China zunehmend 
zum wichtigsten wirtschaftlichen Akteur. Moskau und Peking haben hier bisher eine stillschweigende Arbeitsteilung praktiziert, und beide Staaten bewerten ihre Kooperation als positiv. ${ }^{12}$

Ein Beispiel für diese Zusammenarbeit ist das Wirtschafts- und Handelskooperationsabkommen zwischen China und der Eurasischen Wirtschaftsunion, zu der Kasachstan, Kirgistan, Armenien, Weißrussland und Russland gehören. Das Abkommen ist seit 2019 in Kraft. Es zeigt, dass sich die Beziehungen zwischen China und Russland vertiefen - während sich die Fronten zwischen dem Westen und den beiden Ländern verhärten.

\section{Chinesische Investitionen im Südkaukasus und in Osteuropa}

Die OSZE-Teilnehmerstaaten im Südkaukasus (Armenien, Aserbaidschan und Georgien) und in Osteuropa (Weißrussland, Moldawien und die Ukraine) haben keine gemeinsame Grenze mit China. Und es gibt noch weitere Merkmale, die sie grundsätzlich von Zentralasien und den Staaten des Westbalkans unterscheiden.

Insgesamt sind die Staaten des Südkaukasus und Osteuropas stärker entwickelt als jene Zentralasiens. Im letzten Jahrzehnt waren sie jedoch politisch instabiler als die Länder Zentralasiens und des Westbalkans. Das zeigt etwa der Ukrainekonflikt seit 2014, der Krieg zwischen Armenien und Aserbaidschan im Jahr 2020 und die politische Krise in Weißrussland, die durch die brutale Niederschlagung der Proteste gegen Wahlfälschungen bei den Präsidentschaftswahlen 2020 ausgelöst wurde. Georgien, Moldawien und die Ukraine befinden sich außerdem in einer ständigen politischen und wirtschaftlichen Zerreißprobe zwischen Russland und dem Westen. ${ }^{13}$ Weitere Herausforderungen in dieser Teilregion sind die Korruption sowie die geringe Effizienz der staatlichen Institutionen.

China ist in dieser Teilregion nicht so aktiv wie in Zentralasien. Die Hauptmotive des chinesischen Engagements sind aber auch hier: Transit und Zugang zu Ressourcen.

Entlang der Neuen Eurasischen Landbrücke (New Eurasian Land Bridge) profitiert vor allem Weißrussland von BRIProjekten, da das Land einen unverzichtbaren Transitkorridor entlang der ChinaKasachstan-Russland-Weißrussland-Route in Richtung Europa darstellt. China investiert zudem in weißrussische Industrieprojekte (zum Beispiel in den Great-Stone-Industriepark). Chinesische Banken haben weißrussischen Unternehmen bislang 4,5 Milliarden US-Dollar an Krediten gewährt. ${ }^{14}$

Chinas Investionen in der Ukraine übertreffen bei weitem jenes in Weißrussland, sowohl vor als auch nach der Gründung der „Neuen Seidenstraße“. Fast 70 Prozent der chinesischen Investitionen flossen bereits vor dem offiziellen Start der BRI im Jahr 2013 in die Ukraine. ${ }^{15}$ Bisher legte China den Schwerpunkt auf den Energiesektor (Sonnenenergie) sowie auf die Landwirtschaft (zum Beispiel auf ein neues Getreideexportterminal in Mykolaiv, wodurch die Lieferung nach China erleichtert werden soll). Versuche Chinas, im militärisch-industriellen Bereich der Ukraine Fuß zu fassen, lehnte Kiew 
jedoch bisher immer ab, so auch im Fall von „Motor Sich“, einem Hersteller von Motoren für Militärflugzeuge. Der andauernde Konflikt in der Ostukraine und die damit verbundene soziale, politische und wirtschaftliche Instabilität führen aber zu einer gewissen Zurückhaltung seitens China. ${ }^{16}$ Das Potenzial für chinesische Aktivitäten in der Ukraine ist daher noch nicht ausgeschöpft.

Moldawien ist nur wenig in die "Neue Seidenstraße“ integriert. Das Land liegt am Rande der wichtigsten Wirtschaftskorridore und ist wirtschaftlich kaum entwickelt. Moldawien hat einen vergleichsweise kleinen heimischen Markt und die Wirtschaft ist hauptsächlich landwirtschaftlich geprägt. ${ }^{17}$

Der Südkaukasus bietet China generell nur beschränkte Anbindungsmöglichkeiten. Ein Grund ist, dass es in Georgien bisher keinen Tiefseehafen gibt, obwohl sich China mehrfach darum bemüht hat, in Anaklia einen solchen Hafen zu bauen. ${ }^{18}$

Mit Aserbaidschan hat China jedoch eine Absichtserklärung sowie weitere bilaterale Abkommen unterzeichnet. Dadurch wuchs der bilaterale Handel. AuBerdem investiert China geschätzte 821 Millionen US-Dollar in unterschiedliche Wirtschaftsbereiche jenseits der Ölindustrie. ${ }^{19}$

In Armenien investierte China bisher nur geringfügig. Dies könnte sich jedoch bald ändern. Die beiden Länder haben ein Abkommen über die Entwicklung einer sogenannten Smart City geschlossen. Dafür sollen zwischen 10 und 15 Milliarden US-Dollar in den nächsten 15 Jahren nach Armenien fließen. ${ }^{20}$

\section{Chinas Engagement auf dem Westbalkan}

$\mathrm{Zu}$ den Ländern des westlichen Balkans gehören Albanien, Bosnien-Herzegowina, Kroatien, Kosovo, Montenegro, Nordmazedonien, Serbien und Slowenien. Diese Länder sind wesentlich höher entwickelt als jene Zentralasiens, des Südkaukasus und Osteuropas. In den vergangenen 25 Jahren hat der Westen diese Teilregion stark beeinflusst. Slowenien und Kroatien sind mittlerweile EU-Mitglieder. Doch China beginnt dem Westen in dieser Teilregion zunehmend Konkurrenz zu machen.

Auch auf dem Westbalkan geht es China in erster Linie um den Zugang zu den europäischen Märkten. Deshalb investiert es vor allem in die Infrastruktur der Länder. Der Westbalkan birgt aber auch einige politische und wirtschaftliche Risiken für China. Hierzu zählen Altlasten, die eine Folge des gewaltsamen Zerfalls Jugoslawiens sind, wie etwa anhaltende ethnische Spannungen und schwelende Streitigkeiten um Staatsgrenzen. Hinzu kommen Governance-Probleme wie Korruption und schwächelnde Rechtsstaatlichkeit.

Der Löwenanteil des chinesischen Investments (60 Prozent) entfiel bisher auf Serbien. Chinesische Gelder flossen in den Energiesektor und in den Ausbau der Infrastruktur. Das BRI-Vorzeigeprojekt ist der Ausbau der Bahnstrecke zwischen Belgrad und Budapest. Die Eisenbahnlinie ist eine wichtige Verbindung der „Balkan-Seidenstraße“, welche Budapest, Belgrad, Skopje, Athen und den griechischen Hafen Piräus, der von China betrieben wird, verbindet. Eine ähnli- 
che strategische Bedeutung hat für China auch der Bau einer Autobahn zwischen Belgrad und der südlichen Adria.

In den letzten Jahren haben Serbien und China ihre polizeiliche Zusammenarbeit intensiviert. In Belgrad und anderen serbischen Städten kann man neuerdings beobachten, wie chinesische und serbische Polizisten gemeinsam auf $\mathrm{Pa}$ trouille gehen. Serbien ist das einzige Land, an das China militärische Ausrüstung verkauft hat. Die beiden Länder haben zudem bereits gemeinsame militärische Übungen sowie Anti-Terror-Drills durchgeführt. ${ }^{21}$

Die allermeisten übrigen chinesischen Projekte in den Westbalkanstaaten befassen sich mit dem Ausbau der Verkehrsinfrastruktur. Hier ein paar Beispiele:

- Die Arbër-Autobahn in Albanien, die die Hauptstadt Tirana mit der nordmazedonischen Grenze verbindet. Sie ist Teil des Transportkorridors, der das Ionische Meer mit der bulgarischen Küste des Schwarzen Meeres verbinden soll.

- Die Pelješac-Brücke in Kroatien. Sie wird von der „China Road and Bridge Corporation" gebaut und von der EU mitfinanziert.

- Die Bar-Boljare-Autobahn in Montenegro. Sie verbindet den Hafen Bar im Süden Montenegros mit Serbien. Für den Bau der Autobahn erhielt Montenegro einen Kredit in Höhe von 800 Million Euro von der chinesischen Exim-Bank. Dadurch verschärfte sich die Schuldenkrise des Landes noch weiter.
- Die Kičevo-Ohrid- und MiladinovciŠtip-Autobahnen in Nordmazedonien. Sie liegen direkt an jenem Transportkorridor, der den griechischen Hafen Piräus mit der ungarischen Hauptstadt Budapest verbinden soll.

- Die Integration des slowenischen Hafens Koper in die BRI. Dadurch werden der Schiffsverkehr und der Handel mit der chinesischen NingboZhousan-Hafengruppe gefördert.

Viele chinesische Investitionsverträge und Infrastrukturprojekte in Kroatien und Slowenien verdeutlichen, dass China durchaus gewillt ist, EU-Regeln einzuhalten. Pekings größte Investition in Bosnien-Herzegowina - insgesamt 1,3 Milliarden US-Dollar für den Ausbau des Tuzla-7-Lignite-Kraftwerks und des Stanari-Kraftwerks - widerspricht jedoch den Verpflichtungen Bosnien-Herzegowinas, die EU-Regeln im Umweltschutz einzuhalten.

\section{Wachsender Einfluss Chinas}

Chinas wachsender Einfluss in den drei untersuchten Teilregionen ist offensichtlich. Die „Neue Seidenstraße“ hat Auswirkungen auf die OSZE und ihre 57 Teilnehmerstaaten. Vieles hängt davon $a b$, ob es der OSZE gelingen wird, sich wieder als umfassende und kooperative Sicherheitsorganisation Geltung zu verschaffen. Obwohl sich die Beziehungen des Westens gegenüber China, aber auch gegenüber Russland zunehmend verschlechtern, teilen die OSZE-Staaten mit China ein übergeordnetes Interesse 
an Sicherheit und Stabilität im euroatlantischen und eurasischen Raum. Hier ließe sich ansetzen.

China stellt ein scheinbar attraktives Modell mit einer stabilen Regierungsführung und einer erfolgreichen wirtschaftlichen Einwicklung dar. Das Land hat außerdem bewiesen, dass es gewillt und fähig ist, die globale Ordnung mitzugestalten. ${ }^{22}$ Durch die Umsetzung von Projekten, die dem Wohl der Allgemeinheit dienen, hat China nun einen größeren Anteil an den Prozessen, mit denen die globalen Regeln aufgestellt und durchgesetzt werden. Da die BRI überwiegend einen bilateralen Charakter aufweist, ist sie (noch) kein typisches Integrationsprojekt mit einem eigenen regelbasierten Führungssystem wie etwa die EU oder die Eurasische Wirtschaftsunion. In Zukunft ist es jedoch durchaus möglich, dass sich die BRI in diese Richtung entwickelt und dadurch in Konkurrenz zu bestehenden internationalen Organisationen treten wird.

\section{Chinas umfassendes \\ Sicherheitsverständnis}

China vertritt schon länger ein umfassendes Verständnis von Sicherheit. In einer Rede im Jahr 2014 vor dem vierten Gipfeltreffen der Konferenz für Interaktion und vertrauensbildende Maßnahmen in Asien sprach sich der chinesische Präsident Xi in Shanghai für einen ,gemeinsamen, umfassenden, kooperativen und nachhaltigen" Denkansatz in der Sicherheitspolitik aus. Für Xi bedeutet dies „das Aufrechterhalten der Sicherheit in tradi- tionellen und nicht traditionellen Bereichen“, einschließlich „ethnische und religiöse Probleme [...] Terrorismus, grenzüberschreitende Kriminalität, Sicherheit im Bereich der Umwelt, Cyber-Sicherheit, Sicherheit für Energie und Ressourcen sowie große Naturkatastrophen".23

Diese Vision der Sicherheit ist mittlerweile in der BRI fest verankert: In einem Bericht der ,Leading Group for Promoting the Belt and Road Initiative" (eine Art Kontrollgremium der BRI) aus dem Jahr 2019 ist die oben genannte Rede des chinesischen Staatspräsidenten fast wörtlich wiedergegeben. Der Bericht spricht davon, dass „alle Länder die Vision einer gemeinsamen, umfassenden, kooperativen und nachhaltigen Sicherheit vorantreiben sollen". .24

\section{Bedeutung der Shanghaier Organisation für Zusammenarbeit}

Die gegenwärtigen Zerwürfnisse innerhalb der OSZE könnten dazu führen, dass die Organisation die wichtigsten Aspekte ihres Mandates der umfassenden Sicherheit nicht mehr richtig erfüllen kann. Sollte dieses Szenario eintreten, könnten jene OSZE-Staaten, die bereits enge Beziehungen mit China pflegen (wie etwa die zentralasiatischen Staaten) oder die sich aus geopolitischen oder geoökonomischen Gründen in Richtung Asien orientiert haben (wie etwa Russland), sich der Shanghaier Organisation für Zusammenarbeit (SOZ) zuwenden. Sie könnten die von China angeführte Organisation als eine glaubwürdigere Platt- 
form für die multilaterale Zusammenarbeit mit der BRI ansehen.

Das Mandat der SOZ ist jenem der OSZE sehr ähnlich (wenngleich in der SOZ die Menschenrechtsdimension fehlt). Zusätzlich stellt die SOZ einen multilateralen Mechanismus dar, der die BRI ergänzt und über den Sicherheitsrisiken thematisiert werden können. Im Mai 2017 wurde im Rahmen des OSZE-Forums für Sicherheitskooperation ein Vertrag über günstige Konditionen im Straßenverkehr geschlossen. Der damalige SOZ-Generalsekretär Raschid Alimow stellte dazu fest, dass die SOZ die „legale Basis für gleiche Bedingungen im Straßenverkehr sowie eine einheitliche Plattform für den internationalen Straßenverkehr von Osteuropa in Richtung östliches Russland und China geschaffen hat" ${ }^{25}$ Experten aus China, Russland und Zentralasien betonen bereits seit geraumer Zeit, dass die „Neue SeidenstraBe“ die SOZ auf diese Weise ergänzt. Dies mag auch ein Hinweis darauf sein, dass Russland seinen Widerstand gegen ein umfassenderes Mandat der SOZ allmählich aufgibt und dieses bald realisiert werden kann. ${ }^{26}$

\section{Empfehlungen für eine Kooperation zwischen der OSZE und China}

Die OSZE bietet ein Verhandlungsforum für rivalisierende Bestrebungen. Da die Großmächte weiterhin ihre nationalen Interessen verfolgen und ihre jeweiligen Einflusssphären sichern und erweitern wollen, ist dies wichtiger denn je. Denn diese rivalisierenden Interessen spielen auch eine Rolle innerhalb der OSZE und im Verhältnis der Staaten gegenüber der OSZE. Daher ist es notwendig, China in diese Gespräche einzubeziehen, wenngleich China nicht unbedingt in die OSZE aufgenommen werden sollte. Für die Gespräche mit China müssen die OSZE-Staaten aber eine kohärente Strategie entwerfen und umsetzen.

Für eine konstruktive Zusammenarbeit zwischen der OSZE und China gibt es drei mögliche Hindernisse:

1. Es ist nicht klar, ob die OSZE-Teilnehmerstaaten von einer Kooperation zwischen China und der OSZE profitieren würden. Eine Zusammenarbeit im OSZE-Rahmen könnte die Vorteile schmälern, die durch eine direkte, bilaterale Zusammenarbeit mit China oder eine Kooperation über die EU, die Eurasische Wirtschaftsunion und die SOZ erzielt werden.

2. Eine Zusammenarbeit mit China könnte die OSZE schlicht überfordern. Angesichts der Zerwürfnisse zwischen den OSZE-Teilnehmerstaaten könnte eine Kooperation mit China die OSZE sogar schwächen, insbesondere ihre Fähigkeit, das eigene Mandat umzusetzen und den Konsens über die Normen zu erhalten.

3. Es ist unklar, ob die OSZE für China überhaupt ein attraktives Forum darstellt und unter welchen Umständen Peking eine Kooperation anstreben würde.

Diese Hürden machen eine Zusammenarbeit aber nicht unmöglich. Vielmehr legen sie den Rahmen fest, innerhalb dessen eine Strategie für eine Kooperation mit China entwickelt und umgesetzt 
werden kann. Im Folgenden machen wir Vorschläge, wie sich die OSZE China annähern und mit China und der BRI kooperieren kann.

Schaffung eines Minimalkonsenses über eine Kooperation mit China

Die Durchführungsorgane und Institutionen der OSZE sowie der Vorsitz und die Troika sollten damit beginnen, Zukunftsszenarien für die Beziehungen mit China zu entwerfen. Verschiedene Szenarien können die OSZE-Teilnehmerstaaten für die Auswirkungen der Präsenz und der Aktivitäten Chinas sensibilisieren. Die Staaten können darüber die Herausforderungen und Chancen besser verstehen und auch sehen, welche Handlungsmacht sie haben. Dabei müssen gegensätzliche Ansichten zu China nicht ausgeglichen werden. So ließe sich auf der Basis einer Szenario-Planung ein Minimalkonsens innerhalb der OSZE schaffen, wie eine Kooperation mit China aussehen könnte. Neben der Konsensbildung kann dieses Instrument aber auch für die Politikgestaltung eingesetzt werden.

Verfolgung eines prinzipienfesten, pragmatischen und strategischen Ansatzes gegenüber China

Eine Kooperation sollte über mehrere Kanäle angestrebt werden. Der Dialog ist in Bereichen zu ermöglichen, die von der OSZE als vorrangig eingestuft werden. Gleichzeitig sollte der Kooperations- ansatz aber auch offen für die Anliegen Chinas sein. Daher sind auch Ad-hoc und informelle Formen der Kooperation einzubeziehen.

Ein Kooperationsansatz mit China sollte auf den OSZE-Prinzipien basieren und von einer strategischen Vision geleitet sein, die eine zukünftige formelle Beziehung mit China einschließt. Dafür könnte die OSZE China einen Beobachterstatus einräumen. Es ist denkbar, dass sich dieser allmählich zu einer Partnerschaft entwickelt, die auf Chinas Größe und Bedeutung zugeschnitten ist. Ein möglicher OSZE-Gipfel im Jahr 2025 wäre ein geeignetes Forum, um eine derartige Beziehung mit China zu formalisieren.

\section{Suchen nach Formaten für ein multilaterales Engagement}

Im Rahmen der SOZ könnte die OSZE mit China in Themenbereichen zusammenarbeiten, die von gemeinsamem Interesse sind, etwa der Kampf gegen die organisierte Kriminalität (insbesondere gegen den Drogenhandel), der Schutz kritischer nationaler und grenzüberschreitender Infrastrukturen und die Stabilisierung der Lage in Afghanistan. Die Zusammenarbeit mit anderen internationalen Organisationen, wie etwa der Konferenz für Interaktion und vertrauensbildende Maßnahmen in Asien, wäre ebenfalls möglich. 


\section{Gemeinsames Management der Umweltauswirkungen}

Es könnte sinnvoll sein, einen formellen Dialog mit China über die Bewältigung der Umweltauswirkungen der BRI auf die OSZE-Staaten zu starten. Hier könnte die OSZE ein Forum bieten, in dem gemeinsame Regeln und Prinzipien der Umweltpolitik ausgehandelt werden. Diese Regeln sollten auf internationalen Rahmenverträgen wie der AarhusKonvention aus dem Jahr 1998 beruhen.

\section{Entwicklung einer „Agenda für Konnektivität 2.0“}

Die OSZE, China, die EU und die Eurasische Wirtschaftsunion haben ein unterschiedliches Verständnis von wirtschaftlicher Konnektivität, es gibt aber durchaus Überschneidungen. Damit diese Konnektivitätsvorstellungen trotz ihrer unterschiedlichen normativen Grundlagen zueinander passen und sich auch gegenseitig ergänzen können, empfehlen wir der OSZE, eine „Agenda für Konnektivität 2.0“ zu entwickeln. Diese könnte dazu beitragen, dass sich die Länder nach der Pandemie erholen und die Widerstandsfähigkeit von Volkswirtschaften, Gesellschaften und Institutionen gestärkt wird. ${ }^{27}$ Dies wird nur durch eine gemeinsame Anstrengung all dieser internationalen Akteure möglich sein.

Um den freien Verkehr von Waren, Kapital, Personen und Ideen zu gewährleisten, könnte die OSZE ein eigenes Forum für neue Ansätze und Ideen etablieren. Hierzu kann die OSZE ihre Fä- higkeit, unterschiedliche Akteure zusammenzubringen, sowie ihre Macht, die Tagesordnung zu bestimmen, nutzen. Neben der Bekämpfung protektionistischer Tendenzen sollte es bei diesem Forum auch um effektive und faire nationale und internationale Verwaltungen gehen.

\section{Einbindung Chinas in einen Rahmen für Menschenrechte}

Eine Kooperation mit China im Bereich der Menschenrechte ist derzeit kaum vorstellbar. Chinas jüngste Handlungen sprechen für sich: die anfängliche Vertuschung des Ausbruchs der Corona-Pandemie, die Niederschlagung der Proteste in Hongkong und die Änderungen im Wahlsystem, die Unterdrückung von Menschenrechtsaktivisten sowie die Inhaftierung von Uiguren und anderen turksprachigen Muslimen in Xinjiang.

China weiß, dass fragile Institutionen und eine schwache Rechtsstaatlichkeit die Umsetzung der BRI hemmen können. Es ist sich bewusst, dass soziale und wirtschaftliche Ungleichheiten Missstände schüren, die ihrerseits wieder zu Konflikten und Instabilität führen. Bisher war Chinas Antwort darauf stets: die wirtschaftliche Entwicklung fördern, ohne gleichzeitig eine politische Liberalisierung voranzutreiben. Diese Vorgehensweise Chinas wird sich in Zukunft wohl kaum ändern, das gilt es zu akzeptieren.

Es gibt aber ein gemeinsames Interesse an Stabilität und Sicherheit. Das könnte den Rahmen für eine Zusammenarbeit mit China bilden, in dem auch Menschen- und Minderheitenrechte verankert 
sind. Denn eine Kooperation mit China darf weder die menschliche Dimension der OSZE außer Acht lassen, noch darf sie zu einer Schwächung der menschlichen Dimension im umfassenden Sicherheitsansatz der OSZE führen.

\section{Endnotes}

Ministry of Foreign Affairs of the People's Republic of China, „President Xi Jinping delivers important speech and proposes to build a Silk Road Economic Belt with Central Asian countries", 7 September 2013, https://www.fmprc.gov.cn/mfa _eng/topics_665678/xjpfwzysiesgjtfhshzzf $\overline{\mathrm{h}}$ _665686/t1076334.shtml

National Development and Reform Commission of the People's Republic of China, Ministry of Foreign Affairs of the People's Republic of China, and Ministry of Commerce of the People's Republic of China, "Vision and Actions on Jointly Building Silk Road Economic Belt and 21st-Century Maritime Silk Road.” Beijing: Ministry of Foreign Affairs of the People's Republic of China, 2015, https:/ /www.fmprc.gov.cn/mfa_eng/znnn_66 2805/t1249618.shtml. Accessed 28 July 2021.

3 Communist Party of China, Constitution of the Communist Party of China, Beijing, 24. Oktober 2017, S. 8.

Christoph Nedopil, „Countries of the Belt and Road Initiative (BRI)“, Green Belt and Road Initiative Center, 2021, https://green-bri.org/countries-of-the-b elt-and-road-initiative-bri/

Eine offizielle chinesische Karte der „Belt and Road Initiative" existiert nicht mehr. Die wahrscheinlich umfassendste und genaueste Karte ist auf der Website des Mercator Institute for China Studies abrufbar: „Mapping the Belt and Road Initiative: This is where we stand“, Mercator
Institute for China Studies, 7 June 2018, https://merics.org/en/analysis/mapping-b elt-and-road-initiative-where-we-stand

Yeroen van der Leer/Joshua Yau, „China's new Silk Route: The long and winding road“, PwC, February 2016, p. 4, https://www.pwc.com/gx/en/growth-ma rkets-center/assets/pdf/china-new-silk-r oute.pdf. Weitere Berechnungen des globalen Investments und der Bauverträge Chinas von Derek Scissors zeigen, dass die finanziellen Verpflichtungen Chinas seit 2005 insgesamt über zwei Billionen US-Dollar betragen. Vgl. Derek Scissors, "China global investment tracker", American Enterprise Institute and Heritage Foundation, 2020, https://www.aei.org/ china-global-investment-tracker/

7 Scissors, a. a. O. (Anm. 6).

Dieser Beitrag beruht auf folgendem Bericht: Stefan Wolff, „China's Belt and Road Initiative: Implications for the OSCE“, OSCE Network of Think Tanks and Academic Institutions, March 2021, https://osce-network.net/fileadmin/user _upload/publications/China-BRI-Repo rt-2021-fin.pdf. Der Bericht von Wolff basiert auf 18 Hintergrundpapieren, die bei Expert*innen in Auftrag gegeben wurden. Er bedient sich der einschlägigen wissenschaftlichen Fachliteratur und nutzt Primärdaten aus China, Russland, von der OSZE, der EU, der UNO sowie der Weltbank. Da der Bericht bereits Ende 2020 fertiggestellt wurde, haben wir unsere vorliegende Analyse um die neuesten Entwicklungen ergänzt, zusätzliche Recherchen betrieben und Gespräche mit Fachleuten geführt.

9 OSZE, Gedenkerklärung von Astana: Auf dem Weg zu einer Sicherheitsgemeinschaft, SUM.DOC/1/10/Corr.1*, 3. Dezember 2010, S. 1, https://www.osce .org/mc/74985

10 Assel G. Bitabarova, „Unpacking SinoCentral Asian Engagement along the New Silk Road: A Case Study of 
Kazakhstan“, in: Journal of Contemporary East Asia Studies 2/2018, 149-173; Raffaello Pantucci, „China in Central Asia: The First Strand of the Silk Road Economic Belt", in: Asian Affairs 2/2019, 202-215; Yakov Silin/Larisa Kapustina/Italo Trevisan/Andrei Drevalev, „The Silk Road Economic Belt: Balance of Interests", in: Economic and Political Studies 3/2018, 293-318.

11 Silin et al., a. a. O. (Anm. 10), S. 306.

12 Alexander Gabuev, „Crouching Bear, Hidden Dragon: ,One Belt One Road' and Chinese-Russian Jostling for Power in Central Asia“, in: Journal of Contemporary East Asia Studies 2/2016, 61-78; Baogang He, „The Domestic Politics of the Belt and Road Initiative and its Implications“, in: Journal of Contemporary China 116/2019, 180-195.

13 Tatyana Malyarenko/Stefan Wolff, „The Logic of Competitive Influence-Seeking: Russia, Ukraine, and the Conflict in Donbas", in: Post-Soviet Affairs 4/2018, 191-212.

14 Tatyana Malyarenko, „China's Belt and Road Initiative in the Contested Eastern Neighbourhood“, unveröffentlichtes Papier.

15 Scissors, a. a. O. (Anm. 6).

16 Tatyana Malyarenko, „China's Belt and Road Initiative in Ukraine“, unveröffentlichtes Papier.

17 Malyarenko, a. a. O. (Anm. 14).

18 Giorgi Menabde, „Georgia's Anaklia deep-water port faces a new challenge“, Eurasia Daily Monitor, 18 June 2019, https:/ /jamestown.org/program/georgias-anak lia-deep-water-port-faces-a-new-challeng e/; John C. K. Daly, „Russia’s discreet satisfaction over Georgia's Anaklia port debacle“, Eurasia Daily Monitor, 16 March 2020, https://jamestown.org/program/rus sias-discreet-satisfaction-over-georgias-ana klia-port-debacle/
19 Orkhan Baghirov, „Azerbaijan and China sign $\$ 800$ million economic package“, Eurasia Daily Monitor, 29 May 2019, https://jamestown.org/program/azerbai jan-and-china-sign-800-million-economic -package-the-geo-economic-implications/

20 Nino Kemoklidze, „Armenia, Azerbaijan and China's BRI“, unveröffentlichtes $\mathrm{Pa}$ pier.

21 Mark Webber, „China and the Western Balkans: Opportunities and Challenges for NATO“, unveröffentlichtes Papier.

22 China ist mittlerweile der zweitgrößte Geldgeber für UNO-Friedenseinsätze nach den USA. Der Anteil Chinas am Gesamtbudget der UNO-Friedenseinsätze ist größer als die Beiträge Russlands, Frankreichs und Großbritanniens zusammen. Vgl. UN General Assembly, Implementation of General Assembly Resolutions 55/235 and 55/236, Report of the Secretary-General, Addendum, A/73/350/ Add.1, 24 December 2018, https://und ocs.org/A/73/350/Add.1. Die von China angeführte „Asian Infrastructure and Investment Bank" hat bisher 82 regionale und nicht regionale Mitglieder angeworben und kann Netto-InvestmentVerpflichtungen in Höhe von knapp über acht Milliarden US-Dollar vorweisen. Vgl. Asian Infrastructure Investment Bank, „2019 AIIB Annual Report and Financials", https://www.aiib.org/en/news -events/annual-report/2019/_common/pd f/2019-aiib-annual-report-and-financials.p df

23 Xi Jinping, „Remarks at the fourth summit of the Conference on Interaction and Confidence Building Measures in Asia“, China.org.cn, 21 May 2014, http://www.c hina.org.cn/world/2014-05/28/content_3 2511846.htm

24 Office of the Leading Group for Promoting the Belt and Road Initiative, "The Belt and Road Initiative: Progress, Contributions and Prospects“, Beijing: Foreign Languages Press, 2019, S. 50, https:// 
en.ndrc.gov.cn/newsrelease_8232/202002 /P020200228350122318527.pdf

Rashid Alimov, „Statement at the OSCE Forum for Security Co-operation“, 10 May 2017, p. 3, https://www.osce.org/fi les/f/documents/1/1/316906.pdf

26 Pan Dawei, „SCO and strategic partnership between China and Russia“, Valdai Club, 14 June 2018, https://valdaiclub.com/a/hi ghlights/sco-and-strategic-partnership/?sp hrase_id=902214; Ulugbek Khasanov, "The SCO and the transformation of modern political processes“, Valdai Club, 7 November 2019, https://valdaiclub.com/a/ highlights/sco-and-transformation-moder n-political-processes/?sphrase_id=902214;
Dmitry Suslov, „Non-Western multilateralism: BRICS and the SCO in the postCOVID world“, Valdai Club, 29 July 2020, https://valdaiclub.com/a/highlights/nonwestern-multilateralism-brics-and-the-sco /?sphrase_id=1223135

27 Mit einer „Agenda für Konnektivität 2.0“ meinen wir eine verbesserte Version der derzeitigen OSZE-Konnektivitätsagenda. Vgl. dazu Stefan Wolff, „China: A Challenge or an Opportunity for the OSCE?", Security and Human Rights Monitor, 22 April 2021, https://www.shrmonitor.org/ china-a-challenge-or-an-opportunity-for-t he-osce/ 
